\title{
Thin-wall injection molding of polystyrene parts with coated and uncoated cavities
}

Masato Davide $^{a, b, \dagger}$, Sorgato Marco ${ }^{a}$, Babenko Maksims $^{b}$, Whiteside Ben $^{b}$, Lucchetta Giovanni $^{a}$

${ }^{a}$ Department of Industrial Engineering, University of Padova, via Venezia 1, Padova, Italy

${ }^{\mathrm{b}}$ Faculty of Engineering and Informatics, University of Bradford, Bradford, UK

${ }^{\dagger}$ corresponding author, davide.masato@phd.unipd.it 


\title{
Thin-wall injection molding of polystyrene parts with coated and uncoated cavities
}

Masato Davide, Sorgato Marco, Babenko Maksims, Whiteside Ben, Lucchetta Giovanni

\begin{abstract}
Low-friction mold surface coatings can be used to promote filling of thin-wall parts through reduction of the melt flow resistance by causing wall slip at the polymer-mold interface. This work investigates the effects of different mold coatings (DLC, $\mathrm{CrN}$ and $\mathrm{CrTiNbN}$ ) on the flow resistance of molten polystyrene in thin-wall injection molding. The design of the mold allowed high-speed visualization of the molten polymer flow during the filling phase and measurement of the velocity profile across the cavity thickness. The evaluation of the speed profiles allowed the characterization of the wall-slip phenomenon, indicating the absence of conventional 'fountain-flow' filling mechanism. The results indicate that a DLC deposited on a chrome substrate can significantly reduce the flow resistance of polystyrene, by increasing the slip velocity of polymer melt in contact with the mold surface. Moreover, the contact angle of molten polystyrene over the considered coatings was found to be inversely proportional to the melt flow resistance, indicating the importance of the adhesion at the polymer-mold interface.
\end{abstract}

Keywords: injection molding; wall slip; coatings; flow visualization; wetting 


\section{Abbreviations}

RHCM

PIV

DLC

$\mathrm{CrN}$

$\mathrm{CrTiNbN}$

PA-CVD

$\mathrm{Sa}$
Rapid Heat Cycle Molding

Particle Image Velocimetry

Diamond Like Carbon

Chromium Nitride

Chromium Titanium Niobium Nitride

Plasma Assisted - Chemical Vapour Deposition

Arithmetical mean height 


\section{Introduction}

Reducing the main thickness of injection molded parts leads to material cost savings and lower overall environmental impact (lower resources consumed and improved efficiency). Moreover, as analyzed by Thiriez and Gutowski, the cycle time is shorter for thinner parts making the environmental and economical burdens related to the process less significant too [1]. Thin-wall designs are common in micro-structured components for optical and biomedical applications, such as light guide plates and microfluidic devices. For instance, Masato et al. studied the manufacturing of plastic bio-MEMS for blood depletion characterized by a thin-wall substrate, indicating the relation between the filling mechanism and the replication of micro features [2].

The main feature of thin-wall injection molding is the high flow-length to thickness ratio, which requires a high cavity pressure at the gate during the filling phase to achieve the designed flow length. The polymer viscosity combined with the small cross sectional area of the channel results in a very high-pressure gradient along the component, ultimately hindering the complete replication of mold geometry at the end of flow [3]. In fact, the pressure at the gate is the main factor determining the filling length of a thin-wall cavity [4].

For reduction of the melt flow resistance, different solutions have been considered in the literature, focusing mostly on optimization of the most significant process parameters, such as injection speed and mold temperature [5]. Reduction of the flow resistance has been achieved by means of Rapid Heat Cycle Molding (RHCM), which sustains the mold cavity surface temperature at relatively high temperature during the filling phase, and then reduces the temperature during cooling so the correct ejection temperature can be achieved. De Santis and Pantani suggested that this counteracts the development of the solidified skin layer (which effectively reduces the cross sectional area of the channel) and sustains a lower viscosity of the polymer melt (by reducing cooling rates) [6]. Using RHCM, McFarland et al. molded polystyrene cantilever beams 
characterized by a thickness of $10 \mu \mathrm{m}$ and aspect ratio higher than 170 [7]. Similarly, Chang and Hwang used infrared heating to increase the flow length [8], while Sorgato et al. pursued the same objective using electrical heating and they obtained improvements up to 50\% [9]. As an alternative to RHCM, which requires high initial investments for the mold and complex optimization [10], high-speed injection molding can also be used to allow the polymer melt to completely fill the cavity before the thickening of the skin layer hinders the flow at the core. Masato et al. studied the morphology of a thin-wall cavity (i.e. with a thickness of $350 \mu \mathrm{m}$ ) observing that the extremely reduced thickness results in the absence of the core layer [11]. However, the non-uniform cavity pressure distribution and high shear stresses caused by the high injection speed leads to aesthetic defects, such as flow marks, evidence of polymer degradation and sink marks [12].

Recently, Lucchetta et al. evaluated the effects of mold surface coatings made from low thermal conductivity materials on the reduction of melt flow resistance [13]. Indeed, delaying heat transfer during filling can lead to improvements on the quality of the parts, as shown by Chen et al. for ABS parts molded using TiN and PTFE coatings [14]. These studies provided new insight about the effects of cavity surface coatings on the thermal and tribological boundary conditions at the polymer-mold interface. However, the reduction of the melt flow resistance needs to be thoroughly characterized, especially considering its relationship with the rheology of the polymer melt during the injection phase. Indeed, for thin-wall injection molding the rheological behavior is more complex, due to the effects of pressure [15] and viscoelasticity [16] on polymer viscosity.

According to Zhiltsova et al., the filling of thin-wall injection molding cavities is influenced by the occurrence of the wall-slip effect [17]. In fact, high values of shear rate increase the shear stress at the wall, leading to the disentanglement of the bulk polymer chains [18] and consequently to the unsuitability of the no-slip hydrodynamic boundary condition, which is normally assumed for the 
polymer flow in injection molding. Hatzikiriakos et al. suggested that this effect could be further promoted through the use of hat are generally characterized as 'low friction' [19].

In injection molding, the wall shear stress generally increases with shear rate for a non-slip polymer-wall interface, as indicated by Zhang and Gilchrist [20]. However, Kelly et al. observed that when the shear rate exceeds $10^{7} \mathrm{~s}^{-1}$, the shear stresses can be greater than the critical values leading to deviation from linearity due to the onset of wall-slip [21]. Chien et al. analyzed the cavity pressure drop of polymer melts flowing in injection molding cavities characterized by high shear stress distribution, observing that the wall-slip effect plays a dominant role [22]. Similarly, Chen et al. used a slit flow geometry to evaluate the reduction of the apparent viscosity that follows the onset of the wall-slip effect in thin-wall injection molding [23]. However, both experiments were carried out under isothermal molding conditions (i.e. mold temperature equals melt temperature), which do not apply in injection molding. Moreover, the intensity of the wall-slip phenomenon and of its effect on the melt flow resistance is connected with the properties of the polymer-mold interface [17]. Indeed, according to Yu et al., interface phenomena, such as wall slip, polymer surface tension, roughness and heat transfer, play a fundamental role in thin-wall injection molding that warrants further investigation [24].

In this work, the influence of different mold surface coatings (diamond like carbon, chromium nitride, chromium titanium niobium nitride) on wall slip of polystyrene was experimentally investigated under injection molding process conditions. The filling flow in thin-wall coated cavities was characterized using high-speed particle tracking of the injected polymer. The acquired frames were analyzed by means of Particle Image Velocimetry (PIV) techniques in order to evaluate the slip velocities yielded by the different mold surface coatings. Eventually, the melt flow resistance was related the slip velocity and to the wetting properties of the coatings. 


\section{Materials and methods}

\subsection{Part and mold design}

In this study, the effects of wall slip in thin-wall injection molding were characterized by monitoring the pressure drop of the polymer melt during the filling phase. Two pressure transducers were flush mounted along the flow channel and allowed characterization of the pressure drop.

The mold cavity considered for the investigation is a thin-wall open flow channel, characterized by a length of $40 \mathrm{~mm}$, a width of $6 \mathrm{~mm}$ and a thickness of $0.4 \mathrm{~mm}$. An open-flow cavity was designed in order to sustain the injection of the polymer melt until stationary flow conditions are reached within the cavity. Indeed, the pressure signal after filling the cavity reaches a steady-state value, which represents the equilibrium between the heat convected by the injected polymer melt and that removed by conduction through the mold surface.

The mold assembly was based on a Hasco K-standard modular system. The mold was designed to allow mounting of interchangeable inserts with different coatings, on both fixed and moving halves, as shown in Fig. 1. Several centering elements and coupling units were introduced to guarantee the accurate coupling of the two mold halves upon closing.

In order to characterize the velocity profiles of the melt flow across the channel thickness, the tool allowed the direct visualization of the cavity side during the molding cycle. Two sapphire windows (diameter: $12 \mathrm{~mm}$, thickness: $7 \mathrm{~mm}$ ) were mounted on the moving mold half (Fig. 1) allowing visualization and illumination using a transmission (rather than reflective) configuration.

The mold temperature was set and controlled using two electrical cartridge heaters (diameter: $6.5 \mathrm{~mm}$ ) and a single thermocouple in each of the mold fixed and moving halves. 


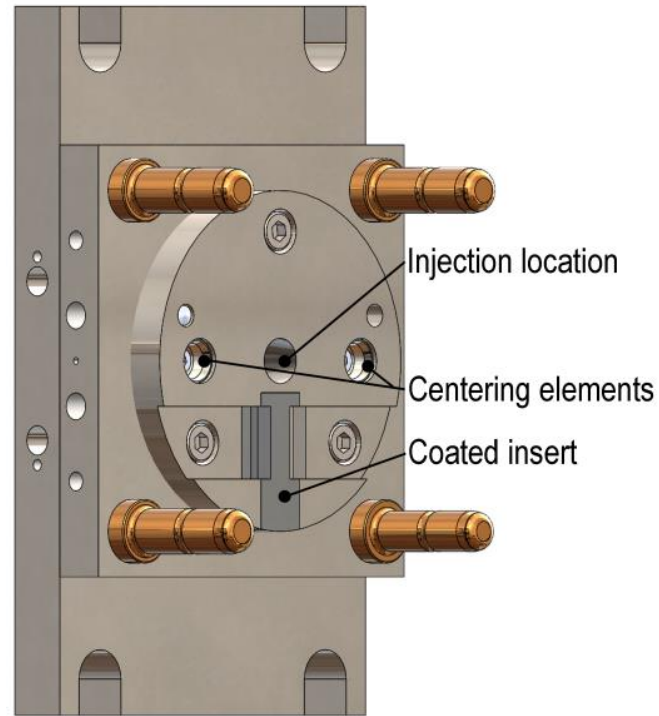

Fix Plate

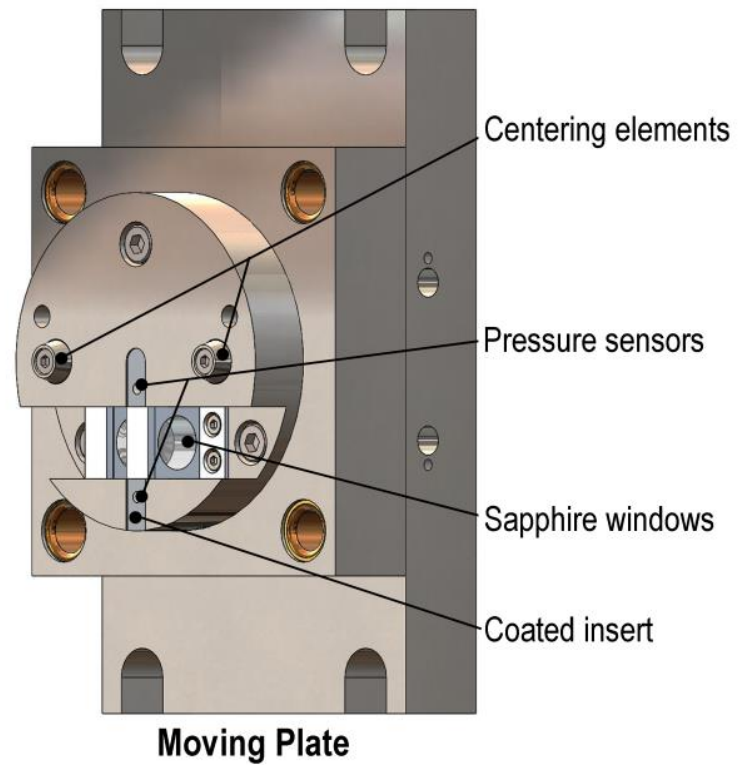

Moving Plate

Fig. 1. Design of the mold inserts holders for the fixed and moving mold halves, respectively.

\subsection{Coating selection}

Three different types of mold surface coatings were selected according to their tribology, hardness and thermal properties: (i) diamond like carbon (DLC), (ii) chromium nitride (CrN) and (iii) chromium titanium niobium nitride $(\mathrm{CrTiNbN})$. All the coatings were deposited by means of Plasma Assisted - Chemical Vapour Deposition (PA-CVD). The use of two distinct adhesion layers (i.e. Cr for DLC v01 and CrN for DLC v02) allowed the deposition of two different DLC coatings. Table 1 reports the main properties of the selected coatings.

Table 1. Main properties of the selected mold surface coatings.

\begin{tabular}{cccccc}
\hline Property & Test Method & DLC v01 & DLC v02 & CrN & CrTiNbN \\
\hline $\begin{array}{c}\text { Coating } \\
\text { thickness [ } \mu \mathrm{m}]\end{array}$ & UNI 1071-2 & $2.0 \pm 0.5$ & $2.0 \pm 0.5$ & $2.5 \pm 0.5$ & $3.0 \pm 0.5$ \\
Adhesion [N] & UNI 1071-3 & $55 \pm 4$ & $55 \pm 4$ & $80 \pm 5$ & $80 \pm 5$ \\
Hardness [HV] & IOS 14577-1 & $2200 \pm 300$ & $2200 \pm 300$ & $2050 \pm 300$ & $2973 \pm 263$ \\
\hline
\end{tabular}




\begin{tabular}{cccccc}
\hline $\begin{array}{c}\text { Friction } \\
\text { coefficient }\end{array}$ & ASTM G99- & $0.15 \pm 0.05$ & $0.15 \pm 0.02$ & $0.60 \pm 0.05$ & $0.65 \pm 0.05$ \\
Adhesion layer & - & $\mathrm{Cr}$ & $\mathrm{CrN}$ & $\mathrm{Cr}$ & $\mathrm{Cr}$ \\
\hline
\end{tabular}

\subsection{Insert characterization}

The surface coatings were deposited onto polished mold inserts $(S a<0.2 \mu \mathrm{m}$ before deposition) made of hardened and tempered H13 chromium-molybdenum steel, having a hardness of approximately $50 \mathrm{HRC}$.

In order to isolate the effects of surface coatings on the melt flow resistance, the surface roughness of the mold inserts was analyzed at five locations over the coated area by means of laser confocal microscopy (Olympus, LEXT OLS4100).

The average values of the surface roughness $(S a)$ evaluated on the different mold inserts were in the range 30 to $50 \mathrm{~nm}$ (Table 2). According to the literature, the effect of surface roughness on the filling flow is significant only below a critical cavity thickness value, which was identified at $150 \mathrm{~mm}[25,26]$. For thicker cavities, the melt flow resistance is not affected by different roughness values of the mold surface. Thus, for the purpose of the current investigation, the topography of the mold inserts was considered comparable and possible effects of surface roughness on the melt flow resistance were neglected.

Table 2. Roughness values for the mold inserts.

\begin{tabular}{lcc}
\hline & \multicolumn{2}{c}{ Sa [nm] } \\
Coating & Avg. Val. & Std. Dev. \\
\hline Uncoated & 41 & 9 \\
DLC v01 & 37 & 8 \\
DLC v02 & 32 & 11 \\
CrN & 43 & 9 \\
CrTiNbN & 46 & 13 \\
\hline
\end{tabular}


The geometry of the coated inserts, assembled into the fixed and moving mold halves, was characterized considering form errors and depth uniformity of the coated channel, in order to avoid any significant geometrical difference among the channels.

\subsection{Material and manufacturing system}

The polymer selected for the injection molding experiments was an amorphous polystyrene (Total, PS Crystal 1540). Table 3 reports the main properties of the polymer.

Table 3. Main properties of the polystyrene used for the molding experiments.

\begin{tabular}{lccc}
\hline Property & Unit & Test Method & Value \\
\hline Density & $\mathrm{g} / \mathrm{cm}^{3}$ & ISO 1183-2:2004 & 1.04 \\
$\begin{array}{l}\text { Melt Flow Index } \\
\left(200^{\circ} \mathrm{C}-5 \mathrm{~kg}\right)\end{array}$ & $\mathrm{g} / 10 \mathrm{~min}$ & ISO 1133-1:2011 & 12 \\
$\mathrm{~T}_{\mathrm{g}}\left(10^{\circ} \mathrm{C} / \mathrm{min}\right)$ & ${ }^{\circ} \mathrm{C}$ & ISO 11357-2:2013 & 100 \\
\hline
\end{tabular}

The injection molding experiments were carried out using a state-of-the-art micro injection molding machine (Wittmann Battenfeld, MicroPower 15) which is characterized by the separation of the metering (a $14 \mathrm{~mm}$ plasticizing screw) and injection systems (a $5 \mathrm{~mm}$ injection plunger). The machine was selected for its performance in terms of maximum injection speed $(750 \mathrm{~mm} / \mathrm{s})$ and maximum injection pressure (2700 bar).

\subsection{Experimental design}

The effects of mold coatings on wall slip and melt flow resistance were analyzed by varying the injection speed from $50 \mathrm{~mm} / \mathrm{s}$ to $750 \mathrm{~mm} / \mathrm{s}$. The other main process parameters were fixed at 
the values reported in Table 4, which were defined considering the literature, recommendations of the materials supplier and the technological limits of the available experimental setup.

In order to achieve an adequate stability of the injection phase, 20 molding cycles were performed before the acquisition of the cavity pressure. For each molding condition 10 acquisitions were collected, one every 5 cycles.

Table 4. Process parameters selected for the injection molding experiments.

\begin{tabular}{lcc}
\hline \multicolumn{1}{c}{ Parameter } & Unit & Value \\
\hline Injection Speed & $\mathrm{mm} / \mathrm{s}$ & $50,100,200,300,450,600,750$ \\
Mold Temperature & ${ }^{\circ} \mathrm{C}$ & 80 \\
Melt Temperature & ${ }^{\circ} \mathrm{C}$ & 240 \\
\hline
\end{tabular}

\subsection{Wetting characterization}

The wetting behavior of the surface coatings to the molten polystyrene was evaluated by measuring the contact angle of a melting cylindrical polymer sample. According to Lee et al., the wetting properties of the coatings were evaluated at the melt temperature adopted for the injection molding experiments (i.e. $240{ }^{\circ} \mathrm{C}$ ) [27]. The experimental setup used for the wettability experiments included electrical heating of an insulated chamber, a light source and a high-speed camera. The tests were performed by (i) heating the chamber up to the test temperature, (ii) inserting the coated surface sample and (iii) recording the wetting behavior of the melting polymer for 10 minutes, as suggested by Sorgato et al. [9]. Images of the melting polymer sample were collected every $90 \mathrm{~s}$ using a $25 \mathrm{X}$ magnification lens. The contour of the melt polymer drop was defined by fitting the base line with a straight line and the curved profile with an ellipse. Then, the left and right contact angles were calculated at the intersection points. 


\section{Process monitoring setup}

\subsection{Cavity pressure measurements}

The effect of mold surface coatings on flow resistance was investigated by monitoring the cavity pressure drop during the injection phase. The pressure was measured in two positions (at 6 $\mathrm{mm}$ and at $30 \mathrm{~mm}$ from the injection location) using Kistler $6182 \mathrm{C}$ piezoelectric pressure transducers.

The piezoelectric charge signal from the sensors was converted into output voltage signal using a Kistler Type 5039A charge amplifier, which was monitored with a National Instruments NI 9205 16-bit analog input module. The acquisitions were performed at a rate of $2500 \mathrm{~s}^{-1}$ (i.e. with a time step of $0.4 \mathrm{~ms})$.

The acquired pressure signals are characterized by a first growth characterizing the filling of the cavity; then, when the first polymer exits from the cavity, the pressure stabilizes to a steadystate value. The pressure measured closer to the injection location increases again at the end of the injection stroke when the velocity of the plunger is decreased for the last few millimeters.

The acquired cavity pressure curves were aligned to the beginning of the injection phase, and the steady-state values of the cavity pressure were collected (Fig. 2). The melt flow resistance was then evaluated by calculating the difference between the average values of the steady-state zones of the two pressure signals (i.e. gate pressure, end pressure). In fact, the difference between the pressure values measured at the two extremities of the channel represents the pressure drop characterizing the mold cavity, which was selected as the response variable for the analysis. 


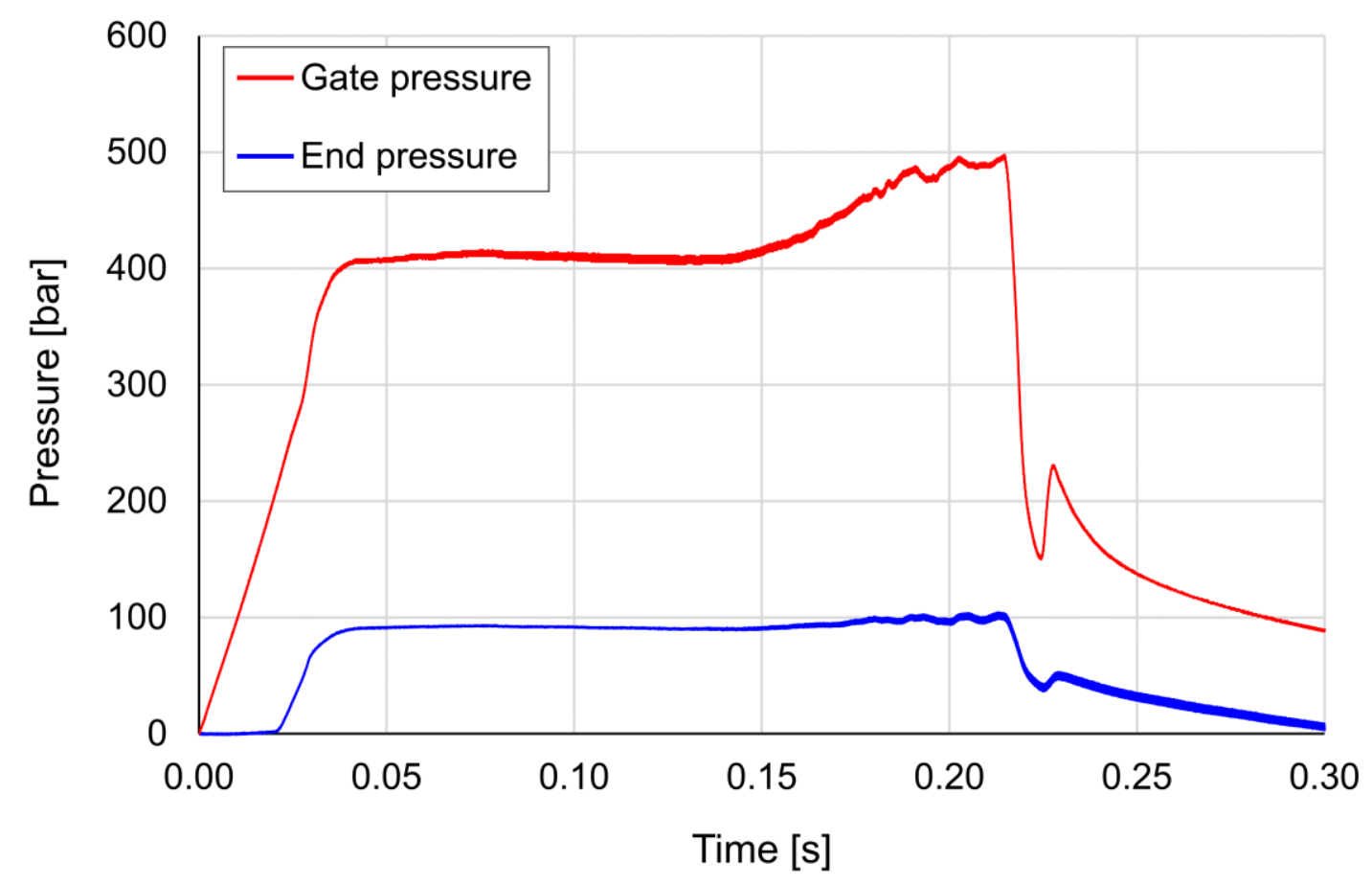

Fig. 2. Pressure evolution measured in the cavity by the two piezoelectric transducers.

\subsection{High-speed flow visualization}

The filling behavior of the polymer in the thin-wall cavity was characterized by direct highspeed flow visualization of the flowing polymer melt on the channel side. The main components and their position in the injection molding machine are shown in Fig. 3 (a). A high-speed camera (NAC, Memrecam HX-3) was aligned to the mold allowing observation through the sapphire window. The online monitoring of the process was performed using a compact telecentric lens $(5 \mathrm{X}$ Mitutoyo) with a 5x magnification and a working distance of $61 \mathrm{~mm}$. The cavity was illuminated by a white light LED illuminator (SugarCUBETM, LED Illuminator) connected to a fiber optic light guide, which was positioned on the other side of the tool, aligned to the camera.

The recording was triggered using a digital signal output from the machine that was synchronous with the initial forward movement of the injection plunger during injection, after the 
mold closed to the viewing position (Fig. 3 (b)). The image sequence was acquired with a frame rate of 12,000 fps, a field of view of $0.53 \mathrm{~mm} \times 3.2 \mathrm{~mm}$ and a resolution of $320 \times 1920$ pixels.

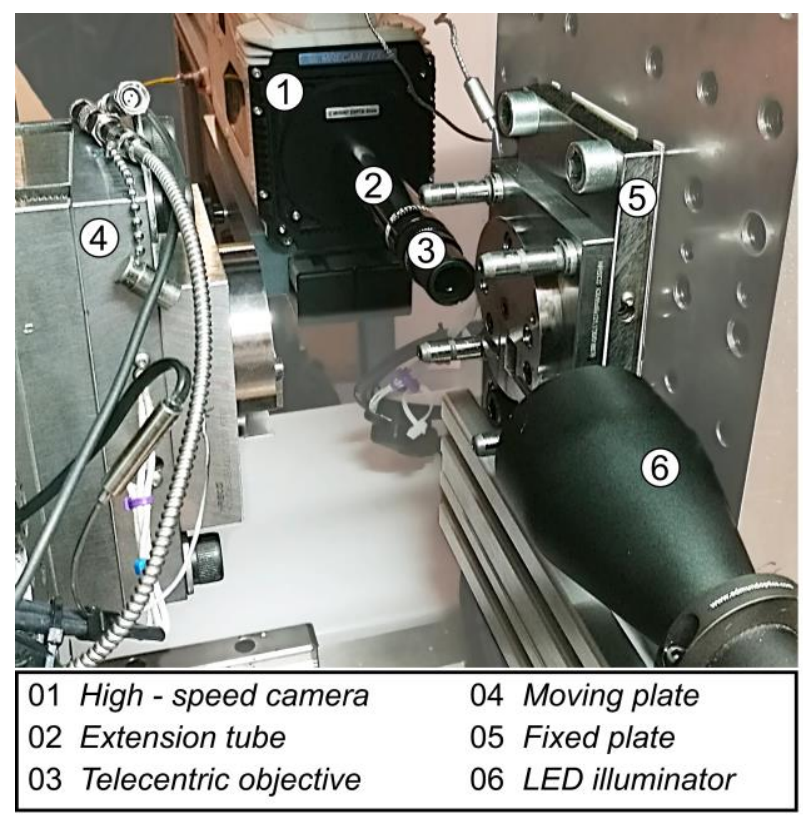

(a)

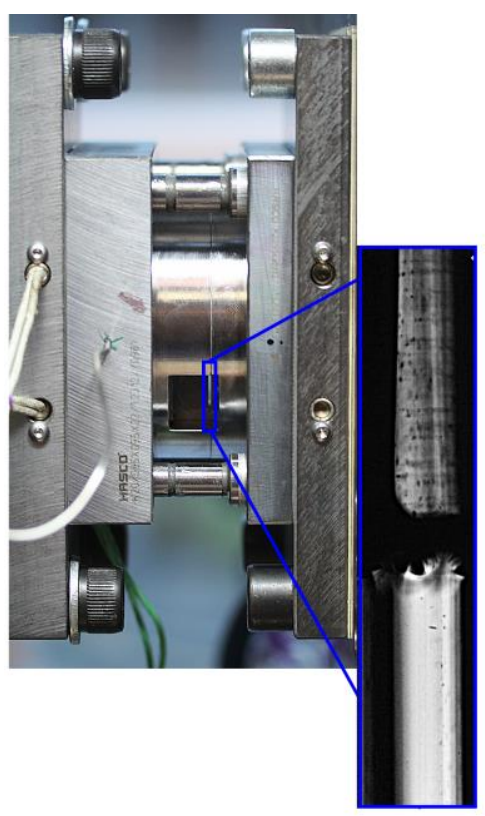

(b)

Fig. 3. Setup used for the high-speed flow visualization of the polymer melt flow, with (a) indication of its main components and (b) detail of the field of view in the closed mold.

\subsection{Particle image velocimetry}

The measurement of the velocity profile of the polymer melt was carried out using Particle Image Velocimetry (PIV), which is an optical method for flow visualization used to perform velocity measurements of flowing fluids [28]. In this work, the PIV analysis of the filling phase was carried out using silica carbide particles (particle size $\sim 17 \mu \mathrm{m}$ ), which were mixed with the polystyrene pellets (1\% weight) in the hopper of the injection molding machine. 
The acquired image sequences (Fig. 4) were processed using the java-based ImageJ software. For each image sequence, acquired for the different coatings at different injection speeds, the coordinates of selected particles were tracked and then plotted against the filling time.

High-speed visualization of the filling flow was carried out in order to:

a) track the velocity profiles of the polymer across the cavity thickness as a function of the set injection speed;

b) evaluate the slip velocity of the polymer melt in the proximity of cavity walls and relate it to the different mold coatings.

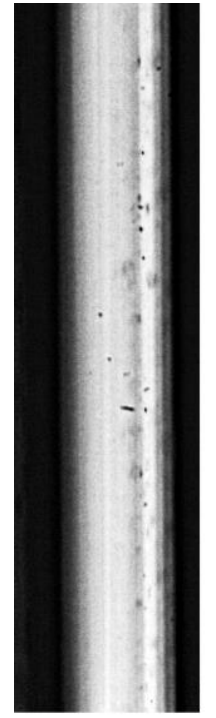

(a)

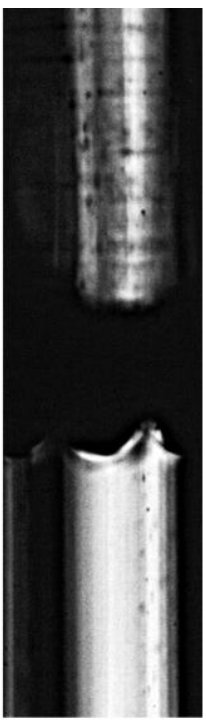

(b)

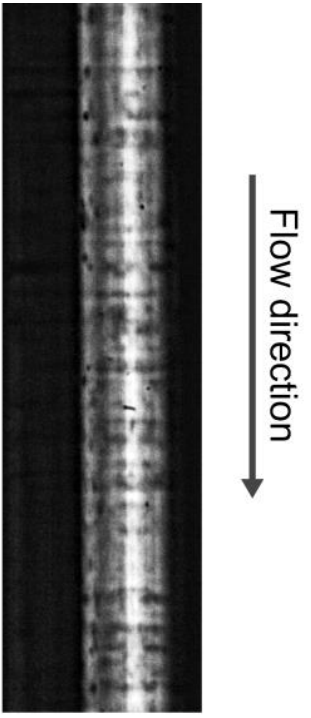

(c)

Fig. 4. Frames of the cavity acquired (a) before the injection (empty cavity), (b) $1.2 \mathrm{~ms}$ after the injection (flow front - view from its side) and (c) $4.7 \mathrm{~ms}$ after the injection (fully developed flow).

\section{Injection molding results}

\subsection{Effect of coatings on flow resistance}


The effects of the selected mold coatings on the flow resistance was analyzed considering the pressure drop in the channel, which was evaluated as the difference between the steady-state values of the pressure signals acquired in the two locations. Fig. 5 shows the dependence of cavity pressure drop on injection speed and mold coating.

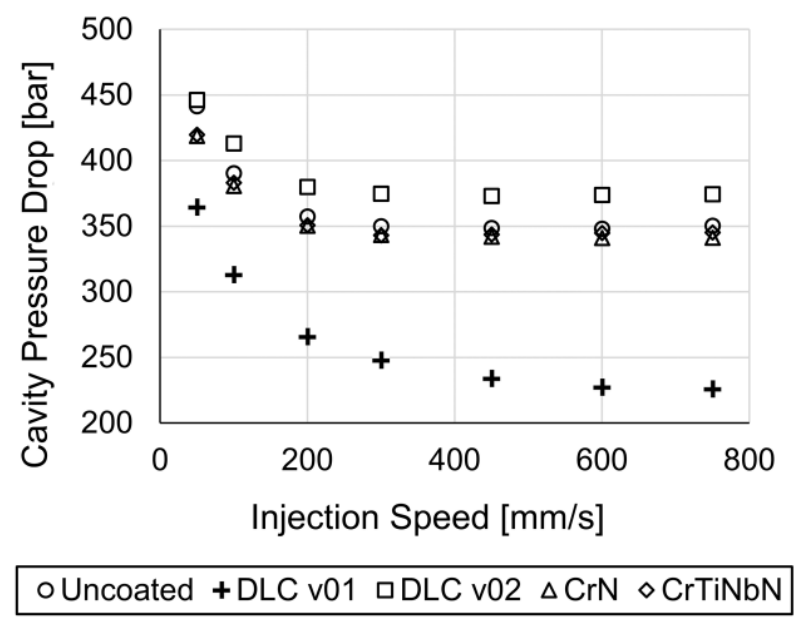

Fig. 5. Experimental measurements of cavity pressure drop as a function of injection speed for the different coatings.

Comparing the experiments performed with the different coatings and the uncoated insert, it is clear that DLC v01 is the coating that most significantly reduces the cavity pressure drop, with an average reduction of about $-28 \%$. The effects of $\mathrm{CrN}(-3 \%)$ and $\mathrm{CrTiNbN}(-2 \%)$ are still positive compared to the uncoated steel but less significant. Conversely, the results obtained with DLC v02 indicated a negative effect on the cavity pressure drop $(+6 \%)$.

For all the tested coatings, the observed effect of increasing the injection speed is to decrease the cavity pressure drop until the achievement of a stabilized value at about $300 \mathrm{~mm} / \mathrm{s}$. In fact, during the injection phase, a thin layer of polymer is adsorbed at the mold surface interface thus creating an insulating layer, whose thickness controls the pressure drop in the cavity. For increasing 
flow rates, the thickness of the skin layers decreases until it reaches a minimum and so does the cavity pressure drop.

The plateau observed at higher injection speed (i.e. from 300 to $750 \mathrm{~mm} / \mathrm{s}$ ) differs from the normal increase that occurs in conventional injection molding. In fact, increasing the flow rate in the cavity does not lead to higher pressure drop, which generally occurs due to the 'fountain-flow' phenomenon [29]. Hence, the unconventional behavior observed for the cavity pressure drop as a function of the flow rate, leads to the hypothesis that the flow conditions are affected by the presence of slipping of the polymer melt occurring in the proximity of cavity walls (i.e. where the shear stress is maximum).

\subsection{Flow instability evaluation}

In injection molding, when there is a non-slip condition at the mold-polymer interface, the shear stress at the wall increases for higher injection speed. However, in thin-wall injection molding the melt flow is characterized by an extremely high value of the wall shear stress, which can lead to the onset of phenomena that are normally neglected for conventional injection molding, such as wall-slip [30].

According to Mhetar and Archer [31], three slip regimes can be distinguished for molten polymers at increasing shear stress: (i) a weak-slip regime beyond a first critical stress $(0.1-0.3$ $\mathrm{MPa}$; (ii) a stick-slip regime with periodic oscillations of the melt flow; (iii) a strong slip regime characterized by stable flow conditions.

The pressure curves acquired in the cavity, especially in the location closer to the gate, are characterized by the presence of periodic vibrations of the signal, as shown in Fig. 6. These oscillations of the plateau-value of cavity pressure indicate that the flow conditions of the injected polymer melt are not stable, thus suggesting the presence of wall-slip. This unstable behavior was 
observed to become more evident for increasingly higher injection speed, indicating the effect of this process parameter on the instability of the flow conditions.
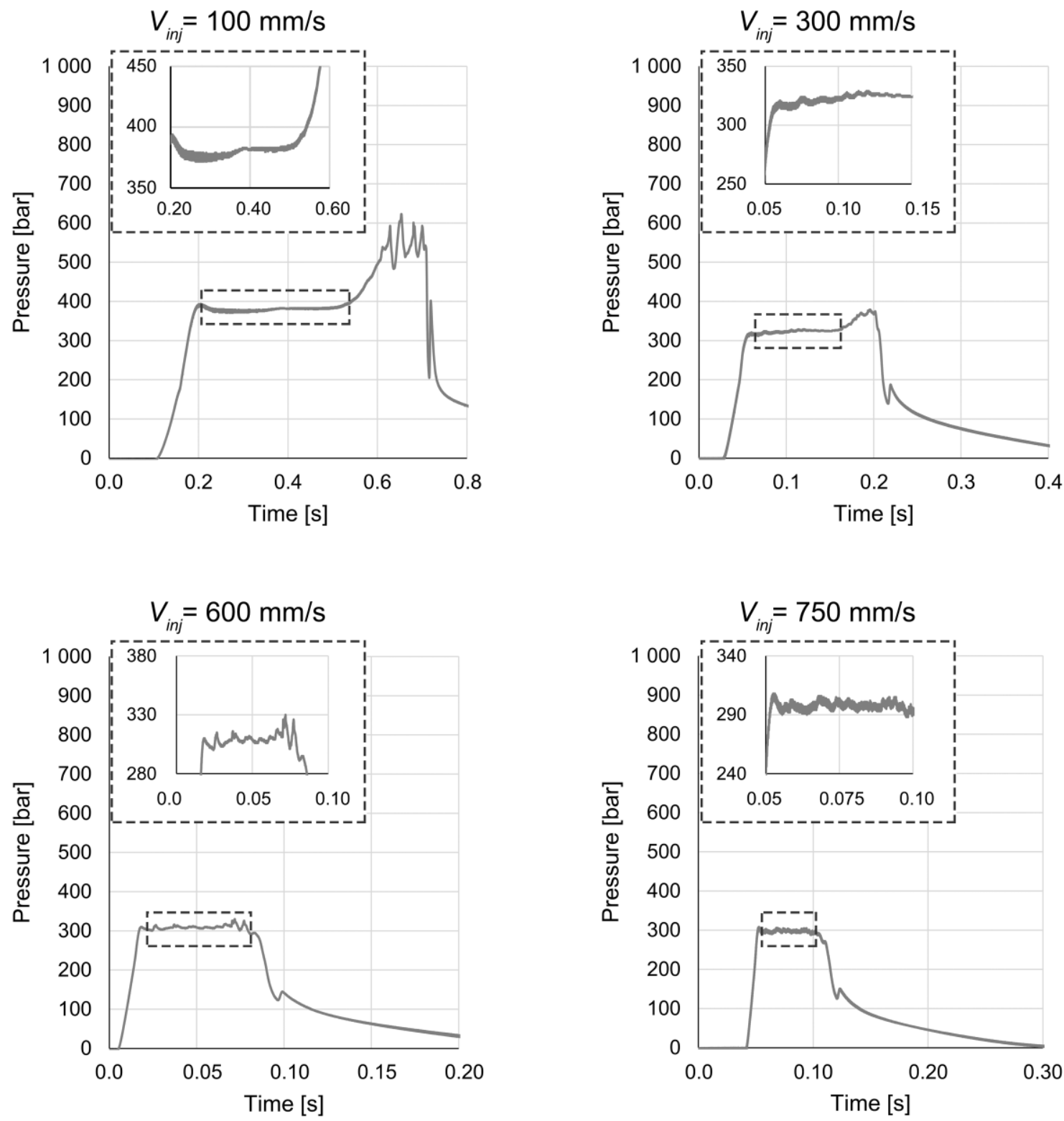

Fig. 6. Cavity pressure curves acquired close to the injection location and evidence of signal oscillation for increasing injection speed. 
Moreover, it was possible to observe that parts molded with injection speed up to $600 \mathrm{~mm} / \mathrm{s}$ were characterized by the presence of small amplitude periodic distortions on their surface, which are related to wall slip [19]. Fig. 7 (a) shows the presence of such defects, also known as sharkskin in polymer extrusion, on the surface of a part injected at $450 \mathrm{~mm} / \mathrm{s}$. For higher injection speed (i.e. $750 \mathrm{~mm} / \mathrm{s}$ ), the melt flow shifts to a strong slip regime characterized by stable conditions. In fact, the surface of the injected polymer is smoother and no sharkskin defects were observed, as shown in Fig. 7 (b).

These observations agree with the findings from Münstedt et al. [32], which identified smooth flow conditions for the extrusion of polyethylene when increasing the shear rate above the stick-slip regime. Moreover, as reported by Rosenbaum et al., when flow conditions are characterized by strong slip the wall shear stress is constant for increasing shear rate [33].

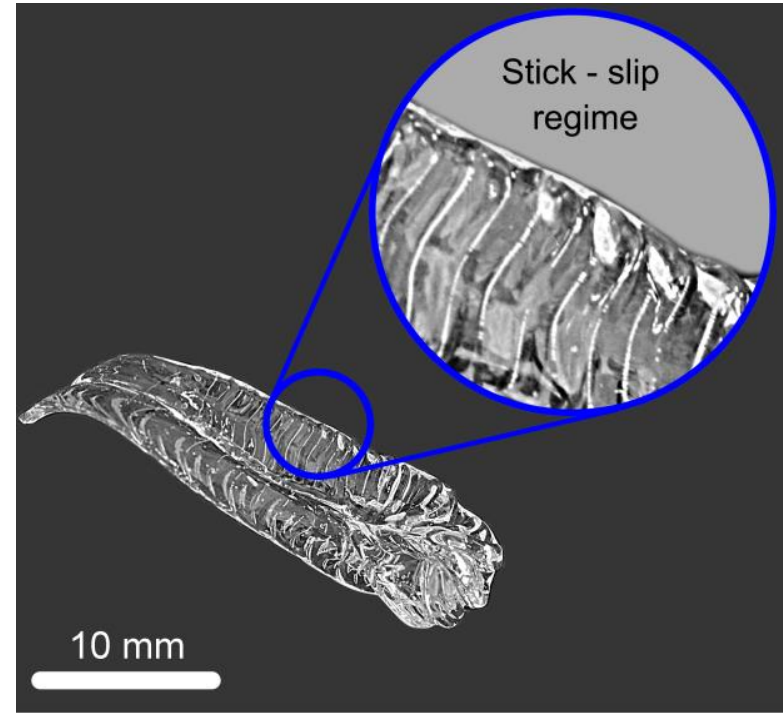

(a)

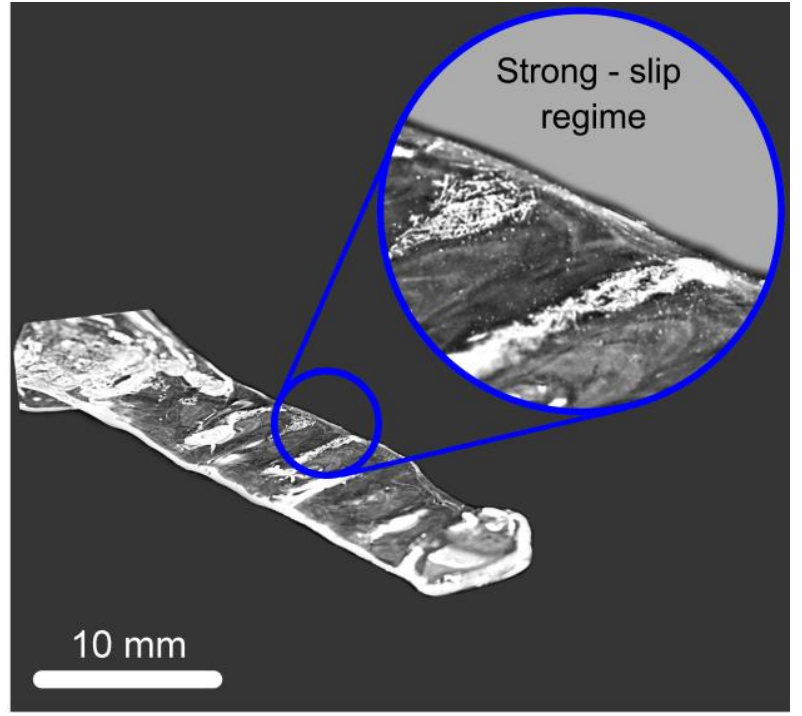

(b)

Fig. 7. Effect of flow instability on polymer injected at (a) $450 \mathrm{~mm} / \mathrm{s}$ and (b) $750 \mathrm{~mm} / \mathrm{s}$ - photo of the polymer flowed from the open-flow channel mold cavity.

\section{Flow visualization results}




\subsection{Characterization of speed profiles}

The filling behavior of the polymer melt was characterized by means of high-speed direct imaging of the flow. The speed profiles of the polymer melt in the uncoated cavity were evaluated during the injection phase by tracking the coordinates of the dispersed silica carbide particles.

For each value of the injection speed, three injection molding tests with flow visualization were carried out. The acquired image frames were analyzed with ImageJ to track the coordinates of selected particles in at least 5 consecutive images. The camera was triggered to the forward movement of the injection plunger and the velocity of the polymer melt was evaluated when the pressure signal had reached its plateau-value.

Fig. 8 reports the results obtained for the coordinates tracking for three repeated injection molding tests executed at an injection speed of $50 \mathrm{~mm} / \mathrm{s}$. The position of the silica carbide particles advances linearly along the channel and the slope of the plot represents the actual polymer speed.

The characterization was performed by exploiting the symmetry of the cavity, and the particles were tracked in only one half of the channel. In order to thoroughly characterize the speed profiles, for each value of the injection speed 5 particles were selected across half of the thickness of the mold cavity. Thus, for each value of the injection speed 15 particles were tracked, for a total of 90 . 


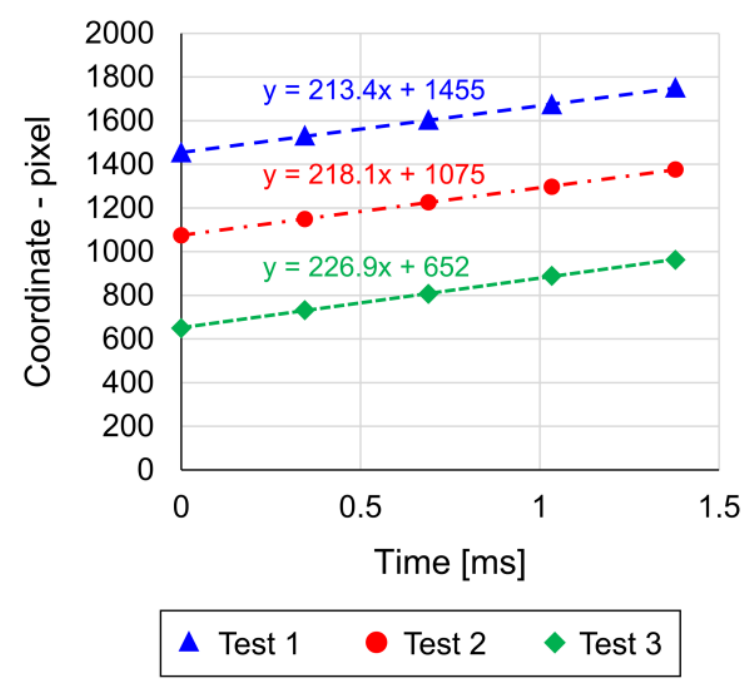

Fig. 8. Coordinate tracking of silica carbide particles in consecutives frames along the cavity (for an injection speed of $50 \mathrm{~mm} / \mathrm{s})$.

The results of the characterization of the speed profiles for the uncoated cavity are reported in Fig. 9, showing the actual polymer speed along the thickness of the cavity for injection speed ranging from 50 to $600 \mathrm{~mm} / \mathrm{s}$.

For all the considered injection speed values, the velocity profiles in the thin-wall cavity are quite flat, which shows that the injected polymer melt is characterized by a plug flow. This proves the presence of wall slip in the thin-wall cavity. Wall slip could be observed for all the values of the injection speed, indicating that the extremely reduced thickness of the cavity produced shear stress at wall that, in the investigated range of process parameters, are always above the threshold for the onset of wall slip.

Two dimensionless numbers, $P e$ and $G z$, were used to evaluate the non-isothermal process that the material experienced during the actual molding process. They all increase with increase of injection speed, as shown by Zhang and Gilchrist [20]. With $2 \times 10^{5}<P e<3 \times 10^{6}$, heat conduction along the flow direction can be safely neglected. 
The Graetz number $(G z)$ varies from 19 to 290; this indicates that heat convection along the flow direction is more important than transverse heat conduction. $G z$ can be used as a measure of the anticipated thickness of the skin layer. Higher values of $G z$ indicate the formation of thinner skin layers. Bay and Tucker suggest that no skin will form if $G z>100$ [34], which corresponds to $v_{i n j}>200 \mathrm{~mm} / \mathrm{s}$ in this study. Therefore the thickness of the skin layer is negligible and the measured pressure stabilizes to a steady-state value (Fig. 2).

Considering the behavior of the cavity pressure drop described in Section 4.1, it can be observed that the thermal effect and the wall slip mechanism both contribute to the cavity pressure drop. Specifically, for low injection speed the cavity pressure drop decreases due the combining effect of a smaller thickness of the skin layers and an increasing slip velocity. Increasing the injection speeds, the thermal insulation effect decreases (cf. reduction of cavity pressure drop shown in Fig. 5 from $50 \mathrm{~mm} / \mathrm{s}$ to $300 \mathrm{~mm} / \mathrm{s}$ ) while the slip velocity is continuously increasing (cf. Fig. 9). In fact, for higher values of the shear rate, the number of macromolecular disentangling continuously increase leading to higher slip velocity, and thus smaller cavity pressure drop.

When the transition to a strong slip regime occurs, the polymer macromolecular suddenly disentangle from a monolayer of polymer chains adsorbed at the polymer-mold interface. Thus, the strong slipping of the polymer is not occurring at a polymer-mold interface, but a polymer-polymer interface [35]. 


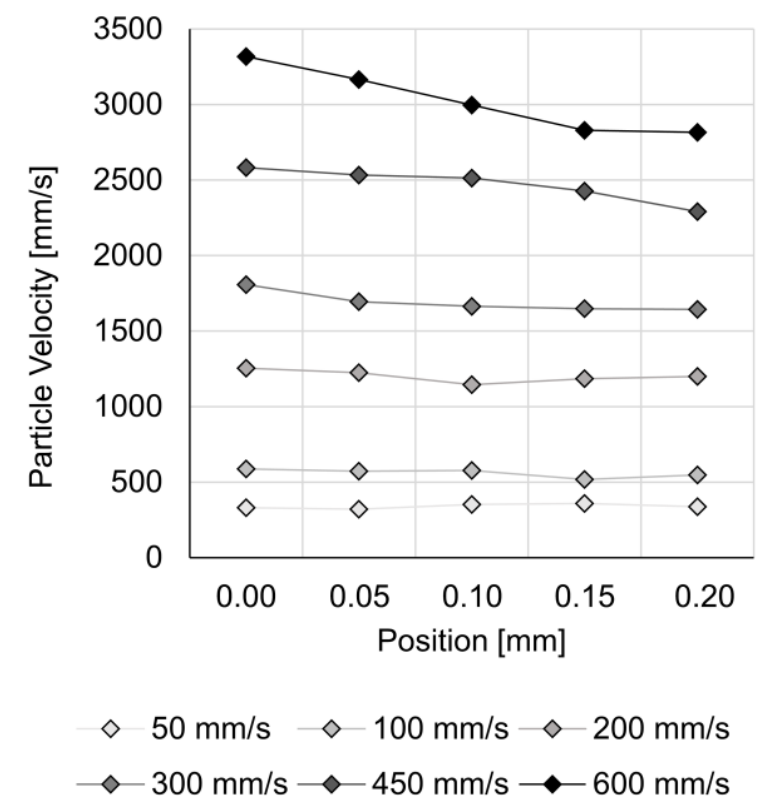

Fig. 9. Velocity profiles for the injected polymer melt with the uncoated insert at different values of the injection speed $(0.00 \mathrm{~mm}$ corresponds to cavity thickness midplane and $0.20 \mathrm{~mm}$ to cavity wall).

\subsection{Slip velocities}

The slip velocity of the polymer melt in contact with mold walls was then characterized for the different mold surface coatings, by tracking the coordinates of the particles as close as possible to the cavity walls. Indeed, due to optical limitations the minimum distance of the particles from the cavity wall was limited to $25 \mu \mathrm{m}$. The analysis was performed for the injection molding experiments carried out with an injection speed of $450 \mathrm{~mm} / \mathrm{s}$.

Table 5 reports the average and standard deviation values of the slip velocity obtained from the PIV analysis of three injection molding replications. The results of the analysis show that the different coatings result in different slip velocities of the polymer melt. In particular, DLC v01 is the coating that resulted in the highest polymer slip velocity at the wall.

Table 5. Slip velocity values for different coatings at an injection speed of $450 \mathrm{~mm} / \mathrm{s}$. 


\begin{tabular}{lcc}
\hline & \multicolumn{2}{c}{ Slip velocity [mm/s] } \\
Coating & Avg. Val. & Std. Dev. \\
\hline Uncoated & 2437 & 79 \\
DLC v01 & 2652 & 52 \\
DLC v02 & 2319 & 153 \\
CrN & 2019 & 209 \\
CrTiNbN & 2267 & 189 \\
\hline
\end{tabular}

The slip velocities were then related to the cavity pressure drop measured in the injection molding experiments carried out with the different coatings (Fig. 10). The plot indicates that cavity pressure drop is inversely dependent on the slip velocity. In fact, coatings that yield low cavity pressure drop are characterized by higher slip velocity. This is particularly evident for DLC v01, which provides the lowest resistance to the flow and has the highest slip velocity.

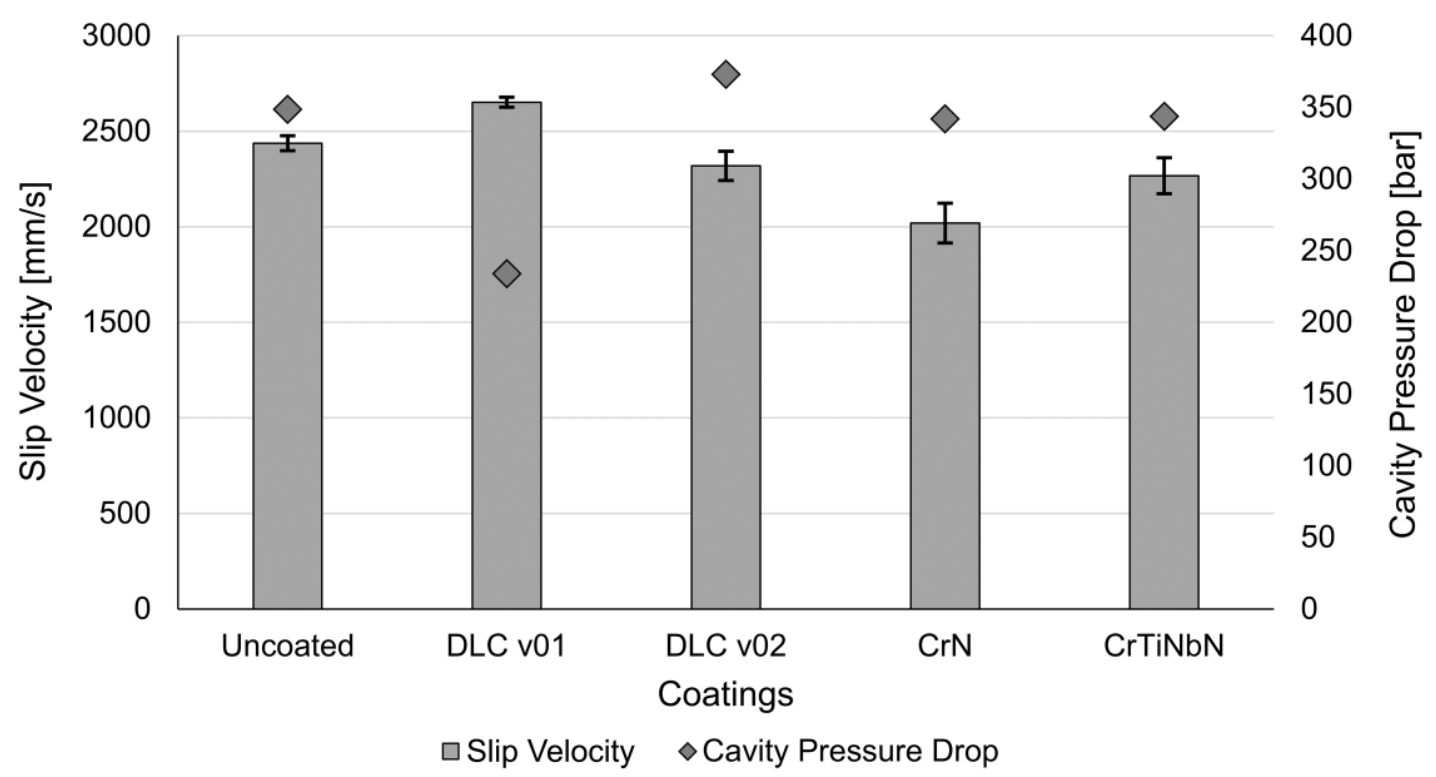

Fig. 10. Relation between slip velocity and cavity pressure drop for the different mold surface coatings for an injection speed of $450 \mathrm{~mm} / \mathrm{s}$.

\section{Wetting behavior}


During the filling phase of the injection molding process, a pressure-supported wetting of the cold mold surface by the molten polymer occurs. Thus, in order to improve the understanding of the effects of the coatings on the melt flow resistance, the wetting behavior of the different coatings to the polystyrene was analyzed. In fact, the wetting properties of the different mold surfaces to the melt polymer can provide information about the intensity of the adhesion at the interface. Indeed, the affinity between each coating-polymer couple is controlled by the different chemistry, as observed by Vera et al. [36].

Table 6 reports the values of the contact angle, indicating that the coatings resulted in diverse wetting behavior due to the different interface interactions.

Table 6. Measurements of the contact angle of polystyrene for the different coatings.

\begin{tabular}{lcc}
\hline & \multicolumn{2}{c}{ Contact Angle $\left[{ }^{\circ}{ }^{\circ}\right.$} \\
Coating & Avg. Val. & Std. Dev. \\
\hline Uncoated & 44.5 & 1.2 \\
DLC v01 & 51.2 & 1.3 \\
DLC v02 & 42.6 & 1.4 \\
CrN & 45.5 & 1.3 \\
CrTiNbN & 46.7 & 1.3 \\
\hline
\end{tabular}

The slip behavior of the injected polymer melt in contact with the coated mold surface is affected by the interactions at the polymer-mold interface. In general, Berger et al. reported that the higher the contact angle the smaller the interactions between the two materials [37]. Indeed, the interfacial tension (i.e. the external stress required to separate a liquid from a solid) is higher for polymer-coating couples characterized by low values of the contact angle. This was proven by correlating the cavity pressure drop, measured during the injection molding process, with the value of the contact angle for each one of the selected coatings. 
The results, reported in Fig. 11, show that the cavity pressure drop is linearly correlated $\left(\mathrm{R}^{2}=\right.$ 0.9121) to the wetting properties measured for each specific polymer-coating interface.

Specifically, the values acquired for cavity pressure drop are inversely proportional to the values of the contact angles. The smaller cavity pressure drop is due to a higher slip velocity, as shown in Fig. 10), which indicates less adhesion of the polystyrene melt to the mold surface upon filling. Hence, the smaller the contact angle is the less adhesion at the interface should be expected during the filling flow. Thus, the wetting behavior of a melt polymer sample over a coated surface can be used to predict the melt flow resistance characterizing the filling phase in thin-wall injection molding.

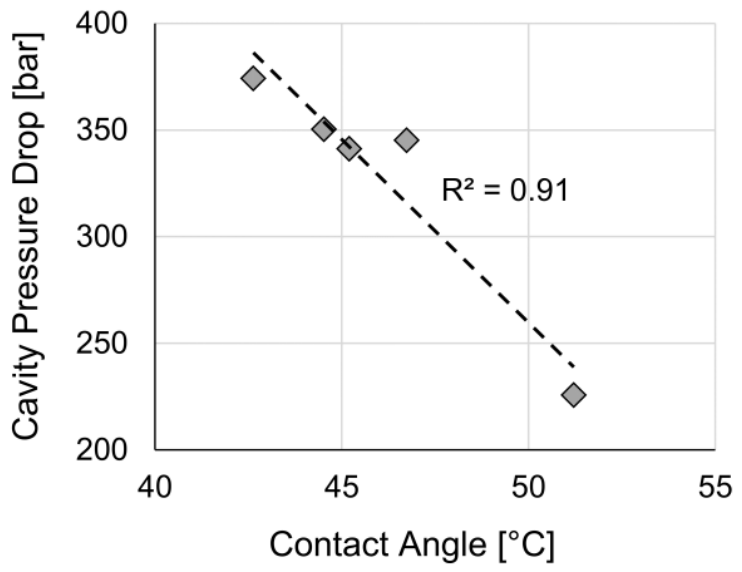

Fig. 11. Correlation between cavity pressure drop and contact angle for the different coatings (injection speed: $750 \mathrm{~mm} / \mathrm{s})$.

\section{Conclusions}

The main objective of this study was the analysis of the filling flow of polystyrene in thin-wall injection molding with coated and uncoated cavities. The mold cavity designed for the injection molding experiments was an open flow channel, with a thickness of $0.4 \mathrm{~mm}$, which allowed the investigation of the wall slip occurring during the filling phase between the hot polystyrene melt and 
the colder mold surface. The effect of mold surface coatings on flow resistance was investigated monitoring cavity pressure drop during the injection phase. Moreover, Particle Image Velocimetry was used to track the speed profiles of the melt flow, thus allowing the characterization of the wall-slip phenomenon.

The results showed that the DLC deposited on chrome substrate (DLC v01) is the coating that most significantly reduces the cavity pressure drop for the selected polystyrene (-28\% compared to the uncoated insert). Limited effects were observed with $\mathrm{CrN}(-3 \%)$ and $\mathrm{CrTiNbN}(-2 \%)$ coatings, while an increase flow resistance (+6\%) was observed with the DLC deposited on chromium nitride (DLC v02).

Considering the effect of injection speed, it was observed that at high injection speed the cavity pressure drop reached a plateau, due to the presence of slipping occurring at the cavity walls. The hypothesis of wall slip was further proved by observing periodic oscillations of the pressure signal. Moreover, the surface of parts molded at injection speed up to $600 \mathrm{~mm} / \mathrm{s}$ showed the presence of small amplitude periodic distortions (sharkskin defect), indicating a stick-slip regime with periodic oscillations of the melt flow. At higher injection speed (i.e. $750 \mathrm{~mm} / \mathrm{s}$ ) the flow shifts to a strong slip regime characterized by stable conditions, as supported also by the absence of sharkskin defects on the surface of the injected polymer.

The filling behavior of the polymer melt was then characterized by means of high-speed visualization of the flow. The velocity profile of the injected polymer was characterized for different values of the injection speed, showing a fully developed plug flow with the presence of a significant wall-slip for both coated and uncoated cavities. In fact, the extremely reduced thickness of the cavity produced shear stress at wall above the threshold for the onset of wall slip.

The measurement of the slip velocities allowed its comparison with the cavity pressure drop for each coating. In particular, it was observed that cavity pressure drop is inversely dependent on the slip velocity, thus indicating the importance of selecting a proper mold surface coating. 
In order to predict the melt flow resistance characterizing the filling phase in thin-wall injection molding, the interface interactions between the polymer and the coatings can be evaluated considering their wetting behavior. The contact angle of a hot polystyrene sample over each coated surface was measured, showing that the melt flow resistance is inversely correlated to the wetting properties. 


\section{Acknowledgements}

The authors gratefully acknowledge the support of Mr. Denis Romagnoli (STS-Group s.r.l., Italy) in the selection and the realization of the mold surface coatings. 


\section{Funding}

This research did not receive any specific grant from funding agencies in the public, commercial, or not-for-profit sectors. 


\section{References}

[1] Thiriez, A., Gutowski, T., 2006. An environmental analysis of injection molding. Proceedings of the 2006 IEEE International Symposium on Electronics and the Environment, 195-200.

[2] Masato, D., Sorgato, M., Lucchetta, G., 2017. Characterization of the micro injection-compression molding process for the replication of high aspect ratio micro-structured surfaces. Microsyst. Technol. 23 (8), 3661-3670.

[3] Lucchetta, G., Sorgato, M., Carmignato, S., Savio, E., 2014. Investigating the technological limits of microinjection molding in replicating high aspect ratio micro-structured surfaces. CIRP Ann.-Manuf. Technol. 63 (1), $521-524$.

[4] Masato, D., Sorgato, M., Lucchetta, G., 2016. Analysis of the influence of part thickness on the replication of micro-structured surfaces by injection molding. Mater. Des. 95, 219-224.

[5] Surace, R., Bellantone, V., Trotta, G., Fassi, I., 2017. Replicating capability investigation of micro features in injection moulding process. J. Manuf. Process. 28, 351-361.

[6] De Santis, F., Pantani, R., 2016. Development of a rapid surface temperature variation system and application to micro-injection molding. J. Mater. Process. Technol. 237, 1-11.

[7] McFarland, A.W., Poggi, M.A., Bottomley, L.A., Colton, J.S., 2004. Injection moulding of high aspect ratio micron-scale thickness polymeric microcantilevers. Nanotechnology, 15, 1628-1632.

[8] Chang, P.C., Hwang, S.J., 2006. Simulation of infrared rapid surface heating for injection molding. Int. J. Heat Mass Transfer 49(21-22):3846-3854.

[9] Sorgato, M., Masato, D., Lucchetta, G., 2017. Effect of vacuum venting and mold wettability on the replication of micro-structured surfaces. Microsyst. Technol. 23 (7), 2543-2552.

[10] Crema, L., Sorgato, M., Lucchetta, G., 2017. Thermal optimization of deterministic porous mold inserts for rapid heat cycle molding. Int. J. Heat Mass Transfer 109, 462-469. 
[11] Masato, D., Rathore, J., Sorgato, M., Carmignato, S., Lucchetta, G., 2017. Analysis of the shrinkage of injection-molded fiber-reinforced thin-wall parts. Mater. Des. 132, 496-504.

[12] Drummer, D., Vetter, K., 2011. Expansion-injection-molding (EIM) by cavity near melt compression About the process characteristic. CIRP J. Manuf. Sci. Technol. 4 (4), 376-381.

[13] Lucchetta, G., Masato, D., Sorgato, M., Crema, L., Savio, E., 2016. Effects of different mould coatings on polymer filling flow in thin-wall injection moulding. CIRP Ann.-Manuf. Technol. 65 (1), 537-540.

[14] Chen, S.C., Chang, Y., Chang, Y.P., Chen, Y.C., Tseng, C.Y., 2009. Effect of cavity surface coating on mold temperature variation and the quality of injection molded parts. Int. Commun. Heat Mass Transfer, 36 (10), $1030-1035$.

[15] Volpe, V., Pantani, R., 2017. Determination of the effect of pressure on viscosity at high shear rates by using an injection molding machine. Journal of Applied Polymer Science.

[16] Kamal, M. R., Goyal, S. K., Chu, E., 1988. Simulation of injection mold filling of viscoelastic polymer with fountain flow. AIChE journal, 34(1), 94-106.

[17] Zhiltsova, T.V., Oliveira, M.S.A., Ferreira, J.A., 2013. Integral approach for production of thermoplastics microparts by injection moulding. J. Mater. Sci. 48 (1), 81-94.

[18] Hatzikiriakos, S. G., 2012. Wall slip of molten polymers. Progress in Polymer Science, 37 (4), 624-643.

[19] Hatzikiriakos, S.G., Stewart, C.W., Dealy, J.M., 1993. Effect of surface coatings on wall slip of LLDPE. Int. Polym. Proc. 8 (1), 30-35.

[20] Zhang, N., Gilchrist, M.D., 2012. Characterization of thermo-rheological behavior of polymer melts during the micro injection moulding process. Polym. Test. 31 (6), 748-758.

[21] Kelly, A.L., Gough, T., Whiteside, B.R., Coates, P.D., 2009. High shear strain rate rheometry of polymer melts. J. Appl. Polym. Sci. 114 (2), 864-873. 
[22] Chien, R.D., Jong, W.R., Chen, S.C., 2005. Study on rheological behavior of polymer melt flowing through micro-channels considering the wall-slip effect. J. Micromech. Microeng. 15 (8), 1389.

[23] Chen, C.S., Chen, S.C., Liaw, W.L., Chien, R.D., 2008. Rheological behavior of POM polymer melt flowing through micro-channels. Eur. Polym. J. 44 (6), 1891-1898.

[24] Yu, L., Koh, C.G., Lee, L.J., Koelling, K.W., Madou, M.J., 2002. Experimental investigation and numerical simulation of injection molding with micro-features. Polym. Eng. Sci. 42 (5), 871-888.

[25] Zhang, H. L., Ong, N. S., Lam, Y. C., 2007. Effects of surface roughness on microinjection molding. Polymer Engineering \& Science, 47(12): 2012-2019.

[26] Bellantone, V., Surace, R., Modica, F., Fassi, I., 2017. Evaluation of mold roughness influence on injected thin micro-cavities. The International Journal of Advanced Manufacturing Technology, 1-11.

[27] Lee, N., Kim, Y.K., Kang, S., 2004. Temperature dependence of anti-adhesion between a stamper with submicron patterns and the polymer in nano-moulding processes. J. Phys. D: Appl. Phys. 37 (12), 1624-1629.

[28] Adrian, R.J., Westerweel, J., 2011. Particle image velocimetry (No. 30). Cambridge University Press.

[29] Greener, J., Wimberger-Friedl, R., 2006. Precision injection molding. Carl Hanser, Munich.

[30] Yang, C., Yin, X.H., Cheng, G.M., 2013. Microinjection molding of microsystem components: new aspects in improving performance. J. Micromech. Microeng. 23 (9), 093001.

[31] Mhetar, V., Archer, L.A., 1998. Slip in entangled polymer melts. 1. General features, Macromol. 31 (24), $8607-8616$.

[32] Münstedt, H., Schmidt, M., Wassner, E., 2000. Stick and slip phenomena during extrusion of polyethylene melts as investigated by laser-Doppler velocimetry. J. Rheol. 44(2): 413-427.

[33] Rosenbaum, E. E., Hatzikiriakos, S. G., Stewart, C. W., 1995. Flow implications in the processing of tetrafluoroethylene/hexafluoropropylene copolymers. Int. Polym. Proc. 10(3): 204-212. 
[34] Bay, R.S., Tucker III, C.L., 1992. Fiber orientation in simple injection moldings. Part 2: Experimental results. Polym. Compos. 13, 332-341.

[35] Ebrahimi, M., Konaganti, V. K., Moradi, S., Doufas, A. K., Hatzikiriakos, S. G., 2016. Slip of polymer melts over micro/nano-patterned metallic surfaces. Soft matter. 12(48): 9759-9768.

[36] Vera, J., Contraires, E., Brulez, A.C., Larochette, M., Valette, S., Benayoun, S., 2017. Wetting of polymer melts on coated and uncoated steel surfaces. Appl. Surf. Sci. 410, 87-98.

[37] Berger, G.R., Steffel, C., Friesenbichler, W., 2016. A study on the role of wetting parameters on friction in injection moulding. Int. J. Mater. Prod. Technol. 52(1-2): 193-211. 


\section{Figures captions}

Figure 1: Design of the mold inserts holders for the fixed and moving mold halves, respectively. Figure 2: Pressure evolution measured in the cavity by the two piezoelectric transducers.

Figure 3: Setup used for the high-speed flow visualization of the polymer melt flow, with (a) indication of its main components and (b) detail of the field of view in the closed mold.

Figure 4: Frames of the cavity acquired (a) before the injection (empty cavity), (b) $1.2 \mathrm{~ms}$ after the injection (flow front - view from its side) and (c) $4.7 \mathrm{~ms}$ after the injection (fully developed flow). Figure 5: Experimental measurements of cavity pressure drop as a function of injection speed for the different coatings.

Figure 6: Cavity pressure curves acquired close to the injection location and evidence of signal oscillation for increasing injection speed.

Figure 7: Effect of flow instability on polymer injected at (a) $450 \mathrm{~mm} / \mathrm{s}$ and (b) $750 \mathrm{~mm} / \mathrm{s}$ - photo of the polymer flowed from the open-flow channel mold cavity.

Figure 8: Coordinate tracking of silica carbide particles in consecutives frames along the cavity (for an injection speed of $50 \mathrm{~mm} / \mathrm{s}$ ).

Figure 9: Velocity profiles for the injected polymer melt with the uncoated insert at different values of the injection speed.

Figure 10: Relation between slip velocity and cavity pressure drop for the different mold surface coatings for an injection speed of $450 \mathrm{~mm} / \mathrm{s}$.

Figure 11: Correlation between cavity pressure drop and contact angle for the different coatings (injection speed: $750 \mathrm{~mm} / \mathrm{s}$ ). 


\section{Tables captions}

Table 1: Main properties of the selected mold surface coatings.

Table 2: Roughness values for the mold inserts.

Table 3: Main properties of the polystyrene used for the molding experiments.

Table 4: Process parameters selected for the injection molding experiments.

Table 5: Slip velocity values for different coatings at an injection speed of $450 \mathrm{~mm} / \mathrm{s}$.

Table 6: Measurements of the contact angle of polystyrene for the different coatings. 